\title{
BMJ Open Comparison of therapy persistence for fixed versus free combination antihypertensives: a retrospective cohort study
}

Thomas Grimmsmann, ${ }^{1,2}$ Wolfgang Himmel ${ }^{3}$

To cite: Grimmsmann T, Himmel W. Comparison of therapy persistence for fixed versus free combination antihypertensives: a retrospective cohort study. BMJ Open 2016;6:e011650. doi:10.1136/bmjopen-2016011650

- Prepublication history for this paper is available online. To view these files please visit the journal online (http://dx.doi.org/10.1136/ bmjopen-2016-011650)

Prior presentations: Preliminary results were presented at the 20th Annual Meeting of the German Drug Utilisation Research Group (GAA), December 2013, Düsseldorf, Germany. http:// www.egms.de/static/en/ meetings/gaa2013/13gaa38. shtml

Received 24 February 2016 Revised 5 August 2016 Accepted 2 September 2016

CrossMark

For numbered affiliations see end of article.

Correspondence to Professor Wolfgang Himmel; whimmel@gwdg.de

\section{ABSTRACT}

Objectives: The aim of the study was to compare therapy persistence among patients who started with one of three drug regimens: a monotherapy, or combination therapy either as a fixed combination (ie, 'single pill') or as a free combination (ie, two separate antihypertensive agents).

Design: In a secondary data analysis, we used descriptive statistics and multivariate logistic regression to measure the effect of the three therapy regimens on therapy persistence over 4 years.

Setting: Prescription data from a large German statutory health insurance provider.

Participants: All patients who started with a new antihypertensive therapy in 2007 or $2008 \quad(n=8032)$ were included and followed for 4 years.

Primary outcome measure: Therapy persistence, defined as receiving a refill prescription no later than within 180 days.

Results: The persistence rates after 4 years were nearly identical among patients who started with a monotherapy $(40.3 \%)$ or a fixed combination of two drugs $(39.8 \%)$. However, significantly more patients who started with free-drug combinations remained therapy persistent $(56.4 \%)$, resulting in an OR of $2.00(95 \% \mathrm{Cl}$ 1.6 to $2.5 ; p<0.0001$ ) for free combinations versus fixed combinations. This trend was observed in all age groups and for men and women. At the end of the study period, the number of different antihypertensive agents was still similar between patients who started with a fixed combination (2.41) and patients who started with a free combination (2.28).

Conclusions: While single-pill combinations make it easier to take different drugs at once, the risk is high that these several substances are stopped at once. Therapy persistence was significantly better for patients who started with a free-drug combination without taking much fewer different antihypertensive drugs as those with a fixed combination.

\section{INTRODUCTION}

Many patients require more than one drug to manage their blood pressure. The assumption

\section{Strengths and limitations of this study}

- In this study, we focus on therapy persistence, not on drug persistence because the main benefits of antihypertensive treatment are largely independent of the drugs employed.

- While guidelines and former studies highlight the advantage of fixed drug combination as a single-pill treatment, we emphasise the risk of stopping several antihypertensive drugs completely when fixed combinations are discontinued.

- Since persistence in hypertension therapy is a long-term issue, a follow-up of 4 years contributed to the validity of the results.

- Owing to the character of our database, it was not possible to determine whether the antihypertensive drugs under observation were exclusively prescribed to treat hypertension. Likewise we cannot definitely conclude that the better persistence results in better clinical outcomes.

is that a simple drug regimen (ie, two drugs combined in a single pill as opposed to two separate pills) improves therapy persistence, one component of adherence. The current European guidelines favour the use of fixeddose combinations of two antihypertensive drugs in a single tablet. ${ }^{1}$ However, this recommendation is based on rather weak evidence, especially two reviews on adherence in hypertensive therapy. One of these reviews is nearly 15 years old, ${ }^{2}$ and the other review was published in $2010^{3}$ but included only two publications that addressed therapy persistence. Those two publications on therapy persistence $^{4} 5$ showed only a small and nonsignificant benefit of fixed combinations compared to separate pills.

A recently published cohort study from Taiwan showed a perplexing result. Many patients who switched from free combinations to a single-pill combination improved in their medication possession ratio, while the adherence of patients who already had 
good adherence to free combinations became worse when they switched to single-pill combinations. ${ }^{6}$

Lack of evidence, small effects and mixing results for single-pill combinations may be the reason why other guidelines, such as the report from the panel members appointed to the Eighth Joint National Committee, ${ }^{7}$ are more flexible and promote the use of separate pills and single-pill combinations for the management of high blood pressure.

The aim of this study was to compare different drug regimens with respect to their effects on therapy persistence. In particular, we compared monotherapy, singlepill combinations of two different drugs (ie, 'fixed combinations') and combinations of two drugs given separately (ie, 'free combinations') for hypertension treatment and hypothesised that fixed combinations improve persistence. We built on a previous study on drug persistence ${ }^{8}$ in which we considered typical patient behaviour including extended drug holidays or irregular repeat prescriptions.

\section{METHODS}

Design

This study was a retrospective cohort study. Patients were followed immediately after starting antihypertensive treatment.

\section{Database}

The database for the study included prescription data for members insured by the Local Health Care funds (AOK) in the federal state of Mecklenburg-West Pomerania. These funds insure $\sim 30 \%$ of the entire population (eg, $\sim 460000$ from 1.6 million people in 2012). The following data were available:

- Pseudonymised identification number of the insured person, including age and sex.

- Central pharmaceutical number-an identification number providing details of the finished drug, including an Anatomical Therapeutic Chemical classification for the active substance and the number of units (tablets, capsules) and defined daily doses (DDD).

- Date of each prescription.

Other details, such as a patient's diagnosis and comorbidities, were not displayed in the database. We followed all patients for whom the general practitioner initiated a new antihypertensive therapy and who received at least two antihypertensive prescriptions in the period of at least 6 years (2160 days) and who received at least two antihypertensive prescriptions, ie, we excluded patients who never filled after their initial prescription. The initiation of therapy was assumed for patients who had no hypertensive treatment for 24 months before the first identified prescription, which resulted in a possible individual follow-up of at least 4 years (1440 days) until December 2012.

\section{Definitions and analysis}

\section{Therapy persistence}

Persistence encompasses the time over which a patient continues to refill a drug prescription and requires a definition of the time periods allowed between refills. ${ }^{9}$ 'Therapy persistence' is the time during which the patient takes any medications, from the initiation of therapy to discontinuation, regardless of the addition of or changes to medication. In contrast, 'medication persistence' is measured at the level of a particular drug. ${ }^{10}$ Evidence indicates that the major drug classes do not differ in their ability to protect against cardiovascular risk caused by hypertension; therefore, therapy persistence is the important factor for assessing cardiovascular risk. Since a prescription in Germany typically covers up to 100 days of treatment, we defined a significant gap in drug coverage as more than 180 days. For our sensitivity analysis, we investigated therapy persistence with a possible gap of up to 360 days.

\section{Antihypertensive drugs}

For the assessment of therapy persistence, we included all antihypertensive agents, as defined by the Anatomical Therapeutic Chemical (ATC) classification; ${ }^{11}$ in particular antihypertensives (ATC code C02); diuretics (C03); $\beta$ blocking agents (C07); calcium channel blockers (C08) and agents acting on the renin-angiotensin system (C09), including fixed-dose combinations with these agents, given the drugs are indicated for the treatment of hypertension.

\section{Drug regimens}

We analysed the initiation of antihypertensive therapy with the following three drug regimens: monotherapy, fixed combinations of two drugs and free combinations of two drugs. To simplify the analysis, patients who started with a combination of three or more different antihypertensive drugs were excluded from further analysis.

\section{Measurement of the repeat prescription interval}

On an individual patient level, we identified the day of the first prescription (day 0) and counted the days between each of the following prescriptions until there were no further prescriptions within 180 or 360 days (figure 1) or until the end of the follow-up period (1440 days after initiation). For each period of interest, we determined the proportion of patients still under therapy. Additional measurements included:

- The total number of prescribed drug substances (ATC codes) per patient 6 months before the initiation of hypertension therapy.

- The number of different hypertension drugs prescribed per patient during each 6-month interval of the study period, as different ATC codes and active substances; these numbers differ in the case of fixed combinations that possess a single ATC code irrespective of the number of the inherent active substances. 


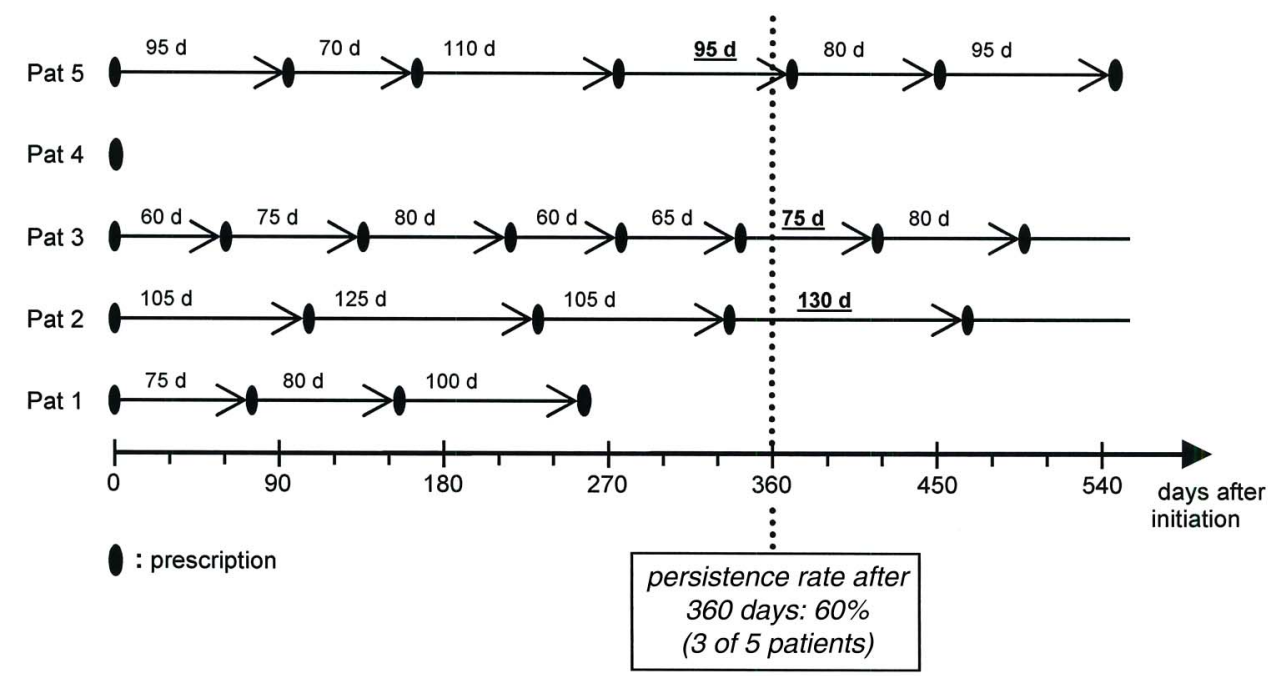

Figure 1 Five repeat prescription scenarios.

- The number and percentage of patients who had continued or discontinued their initial drug regimen after 4 years of therapy persistence.

\section{Statistical analysis}

The main outcomes were the percentage of patients that were therapy persistent after 2,3 and 4 years. The effects of age and gender as modifiers or confounders were investigated in subanalyses. The large sample of patients ensured that even small differences between the three drug regimens became statistically significant, without being clinically relevant. We, therefore, report the confidence intervals (CIs) as a means of how good or precise our estimates are. ${ }^{12}$ Moreover, we calculated the effect of the therapy regime on persistence by a multivariate logistic regression analysis, with ORs and their corresponding 95\% CIs as measures of effect, while controlling for age, gender and number of drugs received before the first prescription of an antihypertensive drug. ${ }^{13}$ The software package SAS V.9.4 was used for statistical analysis.

\section{RESULTS}

\section{Patients and prescriptions}

The prescription histories for a total of 249056 patients in the AOK database could be followed for at least
6 years beginning 1 January 2005, 8032 of these started with a new antihypertensive prescription between 1 January 2007 and 31 December 2008 and received at least a second antihypertensive prescription within 180 days. The majority of these patients $(6502 ; 81.0 \%)$ initially received a monotherapy, $10.8 \%$ received a fixed combination of two drugs and $8.2 \%$ received a free combination of two drugs (table 1). Patients starting with a free combination were somewhat older (62.9 years) than patients starting with a monotherapy (60.0 years) or a fixed combination (60.7 years). More men $(57 \%)$ than women $(43 \%)$ received a fixed combination while the opposite was true for monotherapy and free combinations. In few cases, age and gender were not available. Age was missing for 201 patients starting with a monotherapy, for 26 starting with a fixed combination and for 41 starting with a free combination; gender was missing for 104 patients starting with a monotherapy, 16 starting with a fixed combination and 25 starting with a free combination.

The patients in the three drug regimen groups received a similar number of non-antihypertensive drugs in terms of ATC codes prior to the initiation of hypertension therapy. Specifically, 3.08 (95\% CI 3.01 to 3.14) drugs (ATC codes), on average, were prescribed to patients receiving a monotherapy, 2.76 (2.58 to 2.93) drugs were prescribed to patients receiving fixed

\begin{tabular}{|c|c|c|c|c|}
\hline & Monotherapy $(n=6502)$ & Fixed combination $(n=867)$ & Free combination $(n=663)$ & Total $(n=8032)$ \\
\hline $\begin{array}{l}\text { Age; m (SD) } \\
(95 \% \mathrm{Cl})\end{array}$ & $\begin{array}{l}60.0(15.6) \\
\text { (59.6 to } 60.3)\end{array}$ & $\begin{array}{l}60.7(14.0) \\
(59.7 \text { to } 61.6)\end{array}$ & $\begin{array}{l}62.9(13.3) \\
(61.8 \text { to } 63.9)\end{array}$ & $\begin{array}{l}60.3(15.2) \\
(59.9 \text { to } 60.6)\end{array}$ \\
\hline \multicolumn{5}{|l|}{ Gender } \\
\hline $\begin{array}{l}\text { Male; \% } \\
(95 \% \mathrm{Cl})\end{array}$ & $\begin{array}{l}42.0 \\
(40.8 \text { to } 43.2)\end{array}$ & $\begin{array}{l}45.4 \\
\text { (42.0 to } 48.7 \text { ) }\end{array}$ & $\begin{array}{l}56.6 \\
(52.7 \text { to } 60.4)\end{array}$ & $\begin{array}{l}43.5 \\
\text { (42.4 to } 44.6)\end{array}$ \\
\hline $\begin{array}{l}\text { Female; \% } \\
(95 \% \mathrm{Cl})\end{array}$ & $\begin{array}{l}58.0 \\
\text { (56.8 to } 59.2 \text { ) }\end{array}$ & $\begin{array}{l}54.6 \\
(51.3 \text { to } 58.0)\end{array}$ & $\begin{array}{l}43.4 \\
(39.6 \text { to } 47.3)\end{array}$ & $\begin{array}{l}56.5 \\
(55.4 \text { to } 57.6)\end{array}$ \\
\hline
\end{tabular}


combinations and 2.86 (2.65 to 3.06) drugs were prescribed to patients receiving free combinations with a median of two drugs in each group.

\section{Therapy persistence}

Table 2 shows the persistence rates for the three drug regimens after 1, 2, 3 and 4 years, applying the 180-day and 360-day criteria. With the 180-day criterion for continued drug use (ie, patients received one or more repeat prescriptions with gaps of no more than 180 days), $3342(41.6 \%)$ of the patients were still persistent after 4 years. The groups which started monotherapy and combination therapy were similar: $40.3 \%$ (95\% CI $39.2 \%$ to $41.6 \%$ ) of patients who started with a monotherapy, and $39.8 \%$ (95\% CI $36.5 \%$ to $43.2 \%$ ) of those who started with a fixed combination of two drugs remained therapy persistent. The rate was much higher among patients who started with a free combination of two drugs: $56.4 \%(52.5 \%$ to $60.2 \%)$. In the sensitivity analysis that employed the more liberal criterion of 360-day gaps, the persistence rates were higher, but the relationships among the three groups remained stable (monotherapy: $74.6 \%, 95 \%$ CI $73.6 \%$ to $75.6 \%$; fixed combination: $74.4 \%, 71.6 \%$ to $76.9 \%$; free combination: $82.8 \%, 79.8 \%$ to $85.5 \%)$.

\section{Effects of age, gender and number of previous drugs}

The age of the patient had some influence on therapy persistence. In particular, younger individuals ( $\leq 50$ years) were less persistent. However, the differences in persistence rates among the three drug regimens across the age groups were more important (table 3). Specifically, persistence rates varied by age between monotherapy and fixed combinations, but therapy persistence was highest for patients starting with a free combination therapy in all age groups. Results were similar in the sensitivity analysis that employed the 360-day criterion (data not shown).

Men were somewhat more persistent than women. For example, $44.6 \%$ (95\% CI $39.6 \%$ to $49.7 \%$ ) of the men who started with a fixed combination were persistent after 4 years, while the percentage of women was lower $(35.1 \% ; 30.8 \%$ to $39.6 \%)$. The same tendency could be observed in the case of monotherapy $(41.7 \%$ vs $39.3 \%)$. However, the persistence rates were again much higher for those who were prescribed free combinations at initiation, with $58.7 \%$ of men ( 53.4 to 63.8 ) and $54.5 \%$ of women ( 48.5 to 60.5 ) being persistent after 4 years. This pattern held in the sensitivity analysis that employed the 360-day criterion (data not shown).

There was a slight difference in the number of drugs received before the first prescription of an antihypertensive drug between those patients who were persistent (2.97; $95 \%$ CI 2.88 to 3.06$)$ and those who were not (3.06; 2.98 to 3.14$)$.

In a multivariate analysis, we simultaneously estimated the effect of the therapy regime, age, gender, and number of drugs received before the first prescription of an antihypertensive drug on therapy persistence after 4 years (table 4). Defining 'fixed combination' as reference, the monotherapy group performed similar, with an OR of 1.06 (95\% CI 0.92 to 1.23) while the free combination group performed far superior with an OR of 2.01 (1.63 to 2.49). Gender had a marginal influence, with men performing somewhat better, and younger people performing worse than the older age groups. The number of drugs received before the first prescription of an antihypertensive had only a marginal, non-

Table 2 Therapy persistence for monotherapy, free-drug combinations and fixed combinations

\begin{tabular}{|c|c|c|c|c|c|c|c|c|c|}
\hline \multirow[b]{3}{*}{ Drugs } & \multicolumn{9}{|c|}{ Duration (days of follow-up) } \\
\hline & \multirow{2}{*}{$\begin{array}{l}\text { Initial } \\
\text { Patients } \\
\text { N }\end{array}$} & \multicolumn{4}{|c|}{ Refill prescriptions within 180 days } & \multicolumn{4}{|c|}{ Refill prescriptions within 360 days } \\
\hline & & $\begin{array}{l}360 * \\
(\%)\end{array}$ & $\begin{array}{l}720^{\star} \\
(\%)\end{array}$ & $\begin{array}{l}1080 \\
(\%)\end{array}$ & $\begin{array}{l}1440 \\
(\%)\end{array}$ & $\begin{array}{l}360 \\
(\%)\end{array}$ & $\begin{array}{l}720 \\
(\%)\end{array}$ & $\begin{array}{l}1080 \\
(\%)\end{array}$ & $\begin{array}{l}1440 \\
(\%)\end{array}$ \\
\hline Monotherapy & 6502 & 63.4 & 52.3 & 45.0 & 40.3 & 86.6 & 80.4 & 76.9 & 74.6 \\
\hline Fixed combination of two drugs & 867 & 64.6 & 52.1 & 45.2 & 39.8 & 87.3 & 79.8 & 76.5 & 74.4 \\
\hline Free combination of two drugs & 663 & 78.7 & 69.1 & 62.7 & 56.4 & 92.2 & 88.0 & 85.0 & 82.8 \\
\hline
\end{tabular}

Table 3 Therapy persistence by drug regimen and age after 4 years of follow-up

\begin{tabular}{llll}
\hline Age group & Monotherapy* & Fixed combination* & Free combination* \\
\hline$\leq 50(n=2133)$ & $34.0 \%(31.8 \%$ to $36.3 \%)$ & $38.5 \%(31.9 \%$ to $45.4 \%)$ & $57.0 \%(48.2 \%$ to $65.5 \%)$ \\
$>50-60(n=1686)$ & $42.3 \%(39.7 \%$ to $45.0 \%)$ & $39.3 \%(32.6 \%$ to $46.4 \%)$ & $54.8 \%(46.0 \%$ to $63.4 \%)$ \\
$>60-70(n=1615)$ & $43.6 \%(40.8 \%$ to $46.3 \%)$ & $42.9 \%(35.8 \%$ to $50.3 \%)$ & $58.6 \%(50.2 \%$ to $66.7 \%)$ \\
$>70-80(n=1775)$ & $43.0 \%(40.4 \%$ to $45.6 \%)$ & $38.2 \%(31.0 \%$ to $45.8 \%)$ & $57.7 \%(49.5 \%$ to $65.6 \%)$ \\
$>80(n=555)$ & $42.8 \%(38.2 \%$ to $47.5 \%)$ & $34.0 \%(21.5 \%$ to $48.3 \%)$ & $58.8 \%(44.2 \%$ to $72.4 \%)$ \\
All & $40.3 \%(39.2 \%$ to $41.6 \%)$ & $39.8 \%(36.5 \%$ to $43.2 \%)$ & $56.4 \%(52.5 \%$ to $60.2 \%)$ \\
\hline${ }^{*}$ Percentage and $95 \%$ Cls are reported. & &
\end{tabular}


Table 4 Factors associated with therapy persistence

\begin{tabular}{|c|c|c|c|}
\hline \multirow[b]{2}{*}{ Variable } & \multirow[b]{2}{*}{ Persistence, per cent } & \multicolumn{2}{|c|}{ Effect size (multivariate model) } \\
\hline & & OR (95\% Cl) & p Value \\
\hline \multicolumn{4}{|l|}{ Therapy regimen } \\
\hline Fix combination & 39.8 & 1.0 & \\
\hline Monotherapy & 40.3 & $1.1(0.92$ to 1.23$)$ & 0.4124 \\
\hline Free combination & 56.4 & 2.0 (1.63 to 2.49$)$ & $<0.0001$ \\
\hline \multicolumn{4}{|l|}{ Gender } \\
\hline Female & 39.8 & 1.0 & \\
\hline Male & 43.8 & $1.2(1.06$ to 1.27$)$ & 0.0018 \\
\hline \multicolumn{4}{|l|}{ Age } \\
\hline$\leq 50$ years & 35.9 & 1.0 & \\
\hline$>50-60$ years & 42.9 & 1.3 (1.16 to 1.52$)$ & $<0.0001$ \\
\hline$>60-70$ years & 44.8 & $1.4(1.26$ to 1.65$)$ & $<0.0001$ \\
\hline$>70-80$ years & 43.8 & $1.4(1.24$ to 1.61$)$ & $<0.0001$ \\
\hline$>80$ years & 43.4 & 1.4 (1.17 to 1.72$)$ & 0.0004 \\
\hline Number of drugs & - & 0.99 (0.97 to 1.01$)$ & 0.0992 \\
\hline
\end{tabular}

significant effect on persistence, with more drugs resulting in lower persistence (OR: 0.99; 0.97 to 1.01 ).

\section{Antihypertensive drugs per patient}

In a last step, we investigated the number of antihypertensive drugs (substances) per patient according the different drug regimens and changes over time. This analysis refers only to patients who were persistent over the entire study period ( $\mathrm{n}=3342$ ).

On average, patients who started with a monotherapy and remained persistent for 4 years received 1.57 different antihypertensive drugs within the first 6 months after initiation. At first sight, this figure (1.57) seems to contradict a monotherapy regimen but it only means that the initial drug was changed or a drug was added for about every second patient within these first 6 months. Patients who started with a fixed combination received 2.40 drugs, and patients who started with a free combination received 2.59 drugs (figure 2). The number of drugs increased for patients who started with a monotherapy. They received 1.92 antihypertensive drugs in the last 6 months of observation. The number remained stable for those who started with a fixed combination and decreased a bit for those who received a free combination. However, even after 4 years of observation, the number of different antihypertensive agents was similar between patients who started with a fixed combination $(2.41 ; 95 \%$ CI 2.31 to 2.52$)$ and patients started with a free combination $(2.28 ; 2.19$ to 2.38$)$.

In other words, the number of different antihypertensive agents was still higher for patients starting with a combination therapy, be it a fixed combination or a free combination, than for those who started with a monotherapy. Furthermore, many patients still received the drug regime with which they had started also in the last 6 months of the observation period, $77 \%$ in the case of fixed combinations, $68 \%$ in the case of free combination and $43 \%$ in the case of monotherapy.

\section{DISCUSSION}

\section{Summary of the main findings}

Compared to patients who received a single-pill combination, therapy persistence was significantly better for patients who started with a free-drug combination. This trend was observed in all age groups and for men and women. Throughout the study period, patients who started with a free combination of antihypertensive drugs received nearly the same number of different antihypertensive drugs as those who started with a fixed combination.

\section{Strengths and limitations of the study}

This study took advantage of a large data set that includes every prescription for individuals insured by one major statutory health insurance provider in a large region of Germany. We had the opportunity to study the frequency of repeat prescriptions over a long period of time, which may contribute to the validity of the results because therapy persistence in the context of hypertension is a long-term issue. ${ }^{14}$ A sensitivity analysis also enhanced the internal validity of the results.

Our results could be considered an artefact if a considerable number of patients who started with a free combination of two individual drugs cancelled one of those drugs during the study period. However, the number of antihypertensive drugs per patient starting with a free combination declined only slightly between the start and end of the study period, indicating that the differences in the number of drugs between the three groups remained stable. In other words, patients starting with a monotherapy were less likely to switch to a combination therapy, while those starting with a combination therapy regardless of whether as a fixed or a free combination were more likely to stay on a combination therapy. The number of different antihypertensive substances during follow-up was similar for patients starting with a fixed or a free combination therapy. Furthermore, 
Figure 2 Number of different

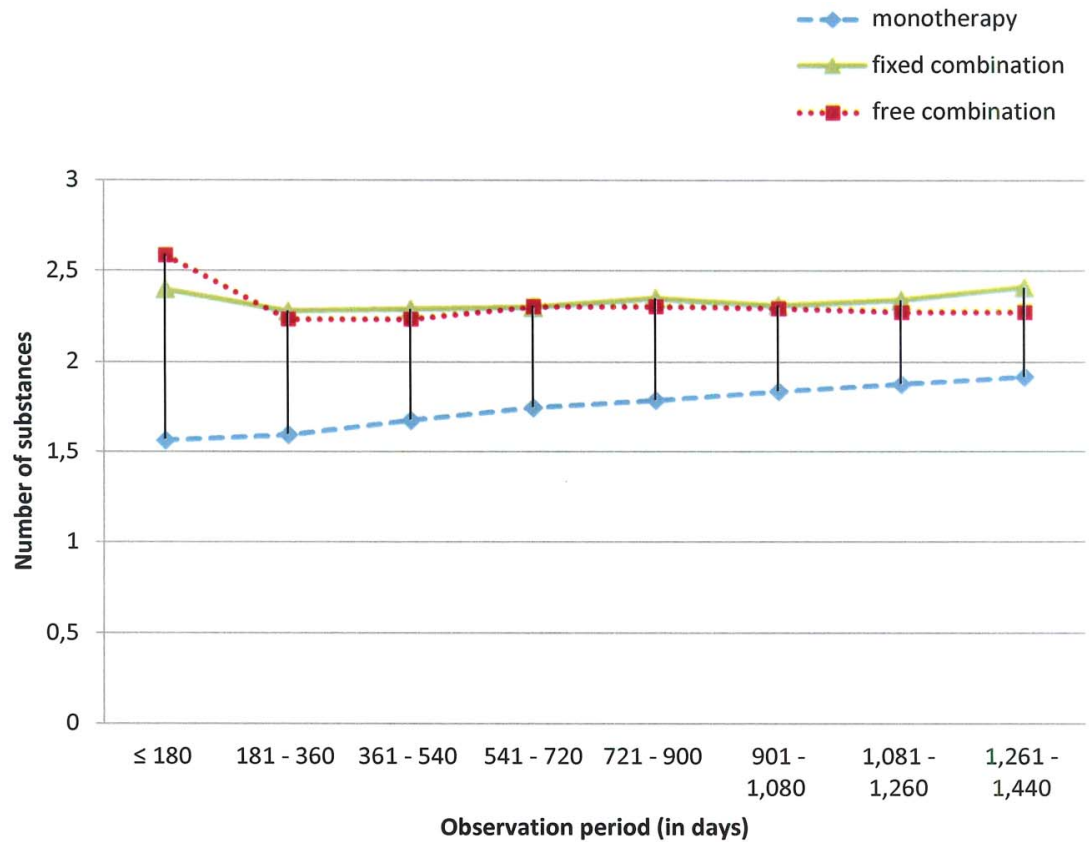

we would like to emphasise that even in such cases when one drug is stopped, patients are better off than those who discontinue both drugs.

The drugs under observation were not necessarily prescribed to treat hypertension, as there are potentially other indications that warrant their use. However, a Norwegian Prescription Database study demonstrated that more than $90 \%$ of users of diuretics and angiotensin II receptor blockers had hypertension. ${ }^{15}$

Our results are based on prescription data only. Therefore, a precondition to recognise the initiation of therapy was that the prescriptions were redeemed so that we missed primary non-adherence of patients who never filled their prescription.

We cannot rule out that physicians selectively prescribed fixed combination drugs to patients who are at higher risk for non-adherence. Although therapy persistence is lower for this group, the use of a fixed combination may have improved persistence beyond what it would have been had patients been prescribed two separate drugs. This effect, ie, 'confounding by indication', could only be avoided by a controlled study in which patients are randomly assigned to receive a fixed or a free combination. However, since comorbidity may influence drug adherence, ${ }^{16}$ and the pill burden before the first prescription of an antihypertensive drug may have motivated the doctor's choice of the drug, we considered the number of drugs received before the first prescription of an antihypertensive drug, as a measure for the pill burden and a proxy for comorbidity, in our regression model. So, it was possible to control, at least, part of the confounding effect.

We should also mention the inherent multilevel structure of our data, ie, patients with certain characteristics, attitudes and behaviour are clustered or nested within physicians or practices with distinct characteristics, philosophy and environment. ${ }^{17}$ So patient behaviour in the same practice may be more similar, compared to individuals in other practices and, vice versa, patients treated by a particular clinician receive care that is influenced by his or her characteristics. Future research should find how much of the variance between the three drug regimens is influenced by significant variation among practices or physicians, after adjusting for patient-level and practice-level covariates.

\section{Comparison with the literature}

Many patients require more than one drug to manage their high blood pressure, and the expectation would be that a fixed-dose combination of two antihypertensive agents in a single tablet would improve drug adherence, especially therapy persistence in comparison to freedrug components given separately. ${ }^{18}$

However, the evidence for this assumption is far from convincing. For example, in a large Italian hypertension study that compared monotherapy and combination therapy, ${ }^{19}$ combination treatment was initially associated with improved therapy persistence; however, no advantage of a fixed-dose combination was observed over time. The authors concluded that the advantage of treatment simplification was less pronounced in their 'real life' study than in studies that focused on this regimen factor alone. In another study, based on newly diagnosed hypertensive patients in German general practices, Hasford $e t a l^{20}$ found that persistence was longest for patients whose initial prescription was for a free combination based on angiotensin-converting enzyme (ACE) inhibitors. In our study, the OR for persistence was significantly higher (2.0) for free combinations, while the OR for monotherapy was nearly the same (1.1) as for fixed combinations. This might be considered evidence for an association between free combination and better 
persistence since, from the patient perspective, monotherapy and fixed combinations are identical, ie, singlepill treatments.

We focused on therapy persistence rather than on drug persistence, because the main benefits of antihypertensive treatment are largely independent of the drugs employed. ${ }^{1}$ This focus on therapy persistence differs from other studies, ${ }^{45}$ and one might even argue that patients starting with free combination have a greater chance of being therapy persistent because they fill their medications on different dates. The better therapy persistence of a free combination would be the result from not taking different hypertensive drugs as agreed on recommended, ie, worse adherence. However, we think we can rule out this possibility, because there was no major difference in the number of antihypertensive agents for 4 years after initiating antihypertensive treatment between the fixed combination and the free combination group.

We observed a gender effect between the three therapeutic regimens, with more men than women starting with a free combination. At first glance, this could be an explanation for the advantage of free combination regimens if we can assume better therapy persistence among men. However, in a review of factors affecting therapeutic compliance, ${ }^{21}$ the results on the relationship between gender and compliance were contradictory. Some studies found female patients to have better compliance, while some studies suggested otherwise; and some found no relationship between gender and compliance. More importantly, we ruled out gender as a confounder and as an explanatory factor by analysing the data separately for gender and could show that men and women had the highest persistence rates if they were prescribed free combinations. We finally confirmed this result by a multivariate analysis, controlling for age and gender and the number of drugs received before the first prescription of an antihypertensive drug, as a measure for the pill burden and a proxy for comorbidity.

Higher costs for fixed combinations might be a reason for a lower persistence if the out of pocket costs are higher, too, for patients and may keep them from regularly filling a prescription. This reason, as discussed by Gradman $e t a l^{22}$ for the USA, does however not seem convincing for German patients, whose out of pocket costs usually range between a minimum of $5 €$ and a maximum of $10 €$ for a single drug. Thus, for regulatory reasons, copayments for a single drug like a fixed combination cannot exceed the copayments for two different drugs like a free combination.

A reason for the prescription of single-pill combinations could be a doctor's motive to reduce the pill burden as Burnier ${ }^{23}$ suggested in his statement on antihypertensive combination treatment. Interestingly, all three patient groups in our study received, on average, nearly the same number of drugs before their physicians initiated an antihypertensive therapy. Obviously, the physicians in our sample did not try to select fixed drug combination according to the previous number of drugs and thus to reduce the pill burden of their patients.

One explanation for our results may be that a free combination of two drugs allows patients to experiment. For example, some patients may prefer to take one pill, and other patients may prefer to have the choice between two pills, allowing them to take the pills at different times or to drop one pill on some days. In their review of qualitative research on lay perspectives on drug adherence, Marshall et $a l^{24}$ found that patients often intentionally adjusted their drug dose, took drugs sporadically and stopped drugs altogether. This behaviour may be triggered by the patient's illness concept and his or her attitudes towards hypertensive drugs and by whether the patient dislikes treatment and fears addiction. ${ }^{24}$

Moreover, while fixed combinations can simplify the treatment regimen by reducing the number of pills, they are accompanied, besides others, by pharmacokinetic threads due to differences in the duration of action of the individual components and less flexibility in modifying the doses of individual components and as a consequence the exposure of patients to unnecessary therapy. ${ }^{25}$

There is a similar explanation in the Taiwan study ${ }^{6}$ why a single-pill combination could worsen medication adherence. Owing to the lack of flexibility with singlepill combinations, the blood pressure may be lowered too much and patients may painfully miss the possibility to adjust drug doses. The feeling of being free to experiment with individual drugs, including the possibility of taking both drugs or cancelling drugs, may correspond to a more responsible role of the patient as his or her own health agent ${ }^{26}$ which may be associated with improved drug persistence. In a more recent study in the $\mathrm{UK},{ }^{27}$ high-risk hypertensive patients were able to master the challenges of self-monitoring and selftitration of antihypertensive treatment and to achieve a larger reduction in blood pressure than control patients who received standard care in which their healthcare clinician adjusted their medication as necessary.

\section{IMPLICATIONS AND CONCLUSIONS}

For patients with markedly high baseline blood pressure, initiation with a combination therapy is recommended. While single-pill combinations make it easier to take different drugs at once, they increase the risk to stop several substances completely. Owing to the high discontinuation rates that occur at the beginning of therapy, ${ }^{8}$ our findings suggest that free combinations may be a good starting point for an antihypertensive therapy.

Considering patient behaviour, simple drug regimens consisting of only one pill with fixed-dose combinations may not represent the most attractive regimens or achieve the best persistence. Future research should focus on patient ideas and attitudes towards different 
drug regimens and determine whether patients prefer to experiment with receiving more than one drug.

\section{Author affiliations}

${ }^{1}$ German Health Insurance Medical Service (MDK) Mecklenburg-Vorpommern, Schwerin, Germany

${ }^{2}$ Department of General Practice, Institute for Community Medicine, University Medicine Greifswald, Greifswald, Germany

${ }^{3}$ Department of General Practice, University Medical Center, Göttingen, Germany

Acknowledgements The authors are indebted to the AOK Nordost for permission to perform this study with special thanks to TG Fiß for his support.

Contributors TG and WH designed the study, performed the statistical analyses, supervised all aspects of the study, wrote the manuscript, critically reviewed and revised the manuscript, approved the final manuscript as submitted and are the guarantors. TG was responsible for the management and retrieval of data.

Competing interests None declared.

Ethics approval Ethical approval was not necessary owing to the nature of the data (secondary data analysis of anonymised files).

Provenance and peer review Not commissioned; externally peer reviewed.

Data sharing statement No additional data are available.

Open Access This is an Open Access article distributed in accordance with the Creative Commons Attribution Non Commercial (CC BY-NC 4.0) license, which permits others to distribute, remix, adapt, build upon this work noncommercially, and license their derivative works on different terms, provided the original work is properly cited and the use is non-commercial. See: http:// creativecommons.org/licenses/by-nc/4.0/

\section{REFERENCES}

1. Mancia G, Fagard R, Narkiewicz K, et al. 2013 ESH/ESC Guidelines for the management of arterial hypertension: the task force for the management of arterial hypertension of the European Society of Hypertension (ESH) and of the European Society of Cardiology (ESC). Eur Heart J 2013;31:1281-357.

2. Claxton AJ, Cramer J, Pierce C. A systematic review of the associations between dose regimens and medication compliance. Clin Ther 2001;23:1296-310.

3. Gupta AK, Arshad S, Poulter NR. Compliance, safety, and effectiveness of fixed-dose combinations of antihypertensive agents: a meta-analysis. Hypertension 2010;55:399-407.

4. Dezii CM. A retrospective study of persistence with single-pill combination therapy vs. concurrent two-pill therapy in patients with hypertension. Manag Care 2001;10(2 Suppl):6-10.

5. Jackson K, Brixner D, Oderda G, et al. Compliance and persistence of fixed dose versus free dose combination therapy with valsartan and HCTZ for patients with hypertension. Value Health 2006;9:A363.

6. Wang TD, Chen $\mathrm{YH}$, Huang $\mathrm{CH}$, et al. Bidirectional adherence changes and associated factors in patients switched from free combinations to equivalent single-pill combinations of antihypertensive drugs. Hypertension 2014:63:958-67.

7. James PA, Oparil S, Carter BL, et al. 2014 evidence-based guideline for the management of high blood pressure in adults: report from the panel members appointed to the Eighth Joint National Committee (JNC 8). JAMA 2014;311:507-20.

8. Grimmsmann T, Himmel W. Persistence of antihypertensive drug use in German primary care: a follow-up study based on pharmacy claims data. Eur J Clin Pharmacol 2014;70: 295-301.

9. Raebel MA, Schmittdiel J, Karter AJ, et al. Standardizing terminology and definitions of medication adherence and persistence in research employing electronic databases. Med Care 2013;51 (Suppl 3):S11-21.

10. Halpern MT, Khan ZM, Schmier JK, et al. Recommendations for evaluating compliance and persistence with hypertension therapy using retrospective data. Hypertension 2006;47:1039-48.

11. WHO Collaborating Centre for Drug Statistics Methodology. Guidelines for ATC classification and DDD assignment 2013. 16th edn. Oslo, 2012, http://www.whocc.no/filearchive/publications/1_ 2013guidelines.pdf (accessed 28 Jun 2015).

12. Altman D, Machin D, Bryant T, eds. Statistics with confidence: confidence intervals and statistical guidelines. 2nd edn. London: BMJ Books, 2000

13. Stokes ME, Davis C, Koch G. Categorical data analysis using SAS. 3rd edn. Cary, NC: SAS Institute, 2012.

14. World Health Organization. Adherence to long-term therapies. Geneva: WHO, 2003:3-93. http://apps.who.int/medicinedocs/pdf/ s4883e/s4883e.pdf (accessed 28 Jun 2015).

15. Selmer R, Blix HS, Landmark K, et al. Choice of initial antihypertensive drugs and persistence of drug use-a 4-year follow-up of 78,453 incident users. Eur J Clin Pharmacol 2012:68:1435-42

16. Calderón-Larrañaga A, Diaz E, Poblador-Plou B, et al. Non-adherence to antihypertensive medication: the role of mental and physical comorbidity. Int J Cardiol 2016;207:310-16.

17. Dickinson LM, Basu A. Multilevel modeling and practice-based research. Ann Fam Med 2005;3(Suppl 1):S52-60.

18. Kuritzky L. Module 4: enhancing adherence with antihypertensives: the role of fixed-dose combinations and home blood pressure monitoring. J Fam Pract 2012;61(8 Suppl):S23-6, S35; quiz S33.

19. Corrao G, Zambon A, Parodi A, et al. Discontinuation of and changes in drug therapy for hypertension among newly-treated patients: a population-based study in Italy. $J$ Hypertens 2008;26:819-24.

20. Hasford J, Schröder-Bernhardi D, Rottenkolber M, et al. Persistence with antihypertensive treatments: results of a 3-year follow-up cohort study. Eur J Clin Pharmacol 2007;63:1055-61.

21. Jin J, Sklar GE, Min Sen Oh V, et al. Factors affecting therapeutic compliance: a review from the patient's perspective. Ther Clin Risk Manag 2008:4:269-86.

22. Gradman $\mathrm{AH}$, Basile JN, Carter BL, et al. Combination therapy in hypertension. J Am Soc Hypertens 2010;4:90-8.

23. Burnier M. Antihypertensive combination treatment: state of the art. Curr Hypertens Rep 2015;17:51.

24. Marshall IJ, Wolfe CD, McKevitt C. Lay perspectives on hypertension and drug adherence: systematic review of qualitative research. BMJ 2012;345:e3953.

25. Angeli F, Reboldi G, Mazzotta G, et al. Fixed-dose combination therapy in hypertension: cons. High Blood Press Cardiovasc Prev 2012;19:51-4

26. Nunes V, Neilson J, O'Flynn N, et al. Clinical guidelines and evidence review for medicines adherence: involving patients in decisions about prescribed medicines and supporting adherence. London: National Collaborating Centre for Primary Care and Royal College of General Practitioners, 2009.

27. McManus RJ, Mant J, Haque M, et al. Effect of self-monitoring and medication self-titration on systolic blood pressure in hypertensive patients at high risk of cardiovascular disease: the TASMIN-SR randomized clinical trial. JAMA 2014;312:799-808. 